\title{
XXVII. On the forms of the vibrations of twitched and stroked strings
}

\section{F. Lindemann}

To cite this article: F. Lindemann (1880) XXVII. On the forms of the vibrations of twitched and stroked strings , Philosophical Magazine Series 5, 9:55, 197-221, DOI: 10.1080/14786448008626826

To link to this article: http://dx.doi.org/10.1080/14786448008626826

册 Published online: 08 Jun 2010.

Submit your article to this journal $\pi$

Џ Article views: 3

Q View related articles $₫$ 
Forms of Vibrations of twitched and stroked Strings. 197

A simplification worth noticing in this process arises from the fact that the digits on the collecting slides, the number of which is always odd, are equal at equal distances from the middle.

12. Since writing the above $I$ have found analogies to the processes described. Lieut.-Col. Oakes, in the Journal of the Institute of Actuaries, Jan. 1863, gives "A method of multiplication which may be practised mentally," the knowledge of which from some other source led me to construct the apparatus. The other processes came, as it were, by the spontaneous action of the machine. Methods having a partial correspondence with them are given by Dr. Peacock in his "Arithmetic" in the Encyc. Met. as Arabian or Persian methods ; also in his 'Algebra,' vol.i. pp. 100-105.

Napier's Promptuarium, described in the Appendix to his Rabdologia, multiplies by a process arithmetically identical with that given ; but the construction and manipulation of his apparatus are very troublesome.

Hampstead, Jan. 2, 1880.

XXVII. On the Forms of the Vibrations of twitched and stroked Strings. By F. LiNDEMANN*.

1. DURING my lectures on Fourier's series and their applications it struck me that various objections might be raised against the theories of twitched and of stroked strings as they are given by Helmholtz $\dagger$. This occasioned the following investigations, which are intended to establish those theories more rigorously.

In regard to the twitched string, the series

$$
y=\frac{2 b \mathrm{~L}^{2}}{\pi^{2} a(\mathrm{~L}-a)} \Sigma \frac{1}{n^{2}} \sin \frac{n \pi a}{\mathrm{~L}} \sin \frac{n \pi x}{\mathrm{~L}} \operatorname{cosin} \frac{2 n \pi t}{\mathrm{~T}}, .
$$

derived in the well-known manner from the general theory, cannot be regarded as the integral of the fundamental differential equation

$$
\frac{\partial^{2} y}{\partial t^{2}}=\alpha^{2} \frac{\partial^{2} y}{\partial x^{2}}, \text { where } \alpha=\frac{2 \mathrm{~L}}{\mathrm{~T}}, . . .
$$

merely because the latter is satisfied by every single term of the series. In the present case the admissibility of this view

* Translated from a separate impression, communicated by the Author, from the Berichte ïber die Verhandlungen der naturforschenden Gesellschaft zu Freiburg i. $B$, vol. vii. no. 4, pp. 500-532.

$\dagger$ Die Lehre von den Tonempfindungen, Brunswick, 1863, Beilage II. and $V$. These supplements remain unaltered in the subsequent editions. 
is much rather to be speeially established * for the twice repeated differentiation of each term of (1) leads to a series which is not convergent. The desired proof is carried out in nos. 2-6. In no. 7 it is shown that the conditions laid down by Christoffel $\dagger$ are fulfilled, under which the occurrence of an acute angle is compatible with the subsistence of the differential equation (2).

The remaining part of this essay is occupied with the stroked string (violin-string). In nos. 8-14, therefore, the form of vibration of the violin-string is discussed without making use of those series. Christoffel's above-mentioned conditions of discontinuity present the necessary means for that purpose. The formulæ found do not fully agree with those of Helmholtz, as is set forth at the end in no. 19; nevertheless the motion of the string proceeds, on the whole, as described by Helmholtz.

As is known, the results thus obtained do not accord with the observations; on this account we have endeavoured, in nos. 15-18, to settle the theory of the stroked string in a more general manner. The results therein gained correspond very well with the observations for the case that the stroking-place is a point marking an aliquot division of the string and lies pretty near one end (distant, at the most, but little more than one fourth of the length of the string from the end, as otherwise node-points are readily formed).

\section{The twitched String.}

2. We will suppose the string to execute transversal vibrations in one plane. In the position of equilibrium let it extend along the positive $\mathrm{X}$ axis from $x=0$ to $x=\mathrm{L}$. Then $\mathrm{L}$ is its length; let $M$ denote its mass, and $P$ the force stretching it; and the form of the string at the time $t$ is determined by the differential equation (2), if M. $a^{2}=\mathrm{L}$. P.

In order to treat the motion of the "twitched string," let us assume that the given string is drawn on one side from its position of rest $y=0$ by means of a pointed peg, while the point of contact of the peg with the string, $x=a$, has moved in the positive direction to the distance $b$ from its resting-place.

* Schlömilch, in his Compendium der höheren Analysis, lays down conditions for the possibility of differentiating geometrical series term by term (2nd edition, vol. ii. p. 139). In the present case those conditions are fulfilled but not applicable; for they imply the presupposition that the differential quotient of the series in question can likewise be represented by a trigonometrical series. But the second differential quotient of (1) is 0 ; and 0 can only be represented by a trigonometric series whose coefficients are collectively $=0$.

†Amali di Matematica, serie 2, t. viii. p. 81. 
At the time $t=0$ the peg is suddenly withdrawn. The initial velocity for every point of the string is then $=0$; and at the time $t=0$ the string has the form of an angularly bent line, whose vertex lies in the point $x=a, y=b$. We have therefore, for $t=0:-$

$$
\left.\begin{array}{l}
y=b \frac{x}{a} \quad \text { for } 0 \leqq x \leqq a ; \\
y=b \frac{\mathrm{L}-x}{\mathrm{~L}-a} \quad, \quad a \leqq x \leqq \mathrm{~L} .
\end{array}\right\} . . . .
$$

If it be admitted that $y$ can be developed as a function of $x$ in a Fourier series of the form

$$
\Sigma\left(\mathrm{A}_{n} \sin \frac{2 n \pi t}{\mathrm{~T}}+\mathrm{B}_{n} \operatorname{cosin} \frac{2 n \pi t}{\mathrm{~T}}\right) \sin \frac{n \pi x}{\mathrm{~L}},
$$

we get, in the known manner, series (1), found also by Helmholtz. For the reason mentioned in no. 1, the admissibility of the latter must, in the present case, be more closely investigated.

3. From D'Alembert's solution of equation (2) it is easily proved that the form of the twitched string consists, at any moment, of three straight lines, apart from single moments in which it is composed of two or only of one line*. Consequently, for all points of the string, $\frac{\partial^{2} y}{\partial x^{2}}=0$; and, consequently also, $\frac{\partial^{2} y}{\partial t^{2}}=0$, by which (2) is fulfilled. We must therefore inquire whether the function $(1)$ is a corresponding linear function of $x$ and $t$.

Of the three right lines, one passes through the initial point, another through the point $x=\mathrm{L}, y=0$. Let these two be cut by the third in the points $\gamma, \delta$ and $\gamma^{\prime}, \delta^{\prime}$ respectively; then is

$$
\left.\begin{array}{lr}
y=\delta \frac{x}{\gamma} & \text { for } 0 \leqq x \leqq \gamma, \\
y=\frac{\gamma \delta^{\prime}-\delta \gamma^{\prime}+\left(\delta-\delta^{\prime}\right) x}{\gamma-\gamma^{\prime}} & , \gamma \leqq x \leqq \gamma^{\prime}, \\
y=\delta^{\prime} \frac{\mathrm{L}-x}{\overline{\mathrm{L}}-\gamma^{\prime}} & , \gamma^{\prime} \leqq x \leqq \mathrm{~L} .
\end{array}\right\} .
$$

* Compare Thomas Young's "Experiments and Inquiries respecting Sound and Light," Phil. Trans. 1800, part i. p. 135; Monge, Journal de l'Ecole Polytechnique, t. viii. p. 118 (1809); and Helmholtz l. c.. 
From this results

$$
\begin{aligned}
\int_{0}^{\mathrm{L}} y \sin \frac{n \pi x}{\mathrm{~L}} d x=\frac{\mathrm{L}^{2}}{n^{2} \pi^{2}}\left[\left(\frac{\delta}{\gamma}-\right.\right. & \left.\frac{\delta-\delta^{\prime}}{\gamma-\gamma^{\prime}}\right) \sin \frac{n \pi \lambda}{\overline{\mathrm{L}}} \\
& +\left(\frac{\delta-\delta^{\prime}}{\gamma-\gamma^{\prime}}+\frac{\delta^{\prime}}{\mathrm{L}-\gamma^{\prime}} \sin \frac{n \pi \gamma^{\prime}}{\mathrm{L}}\right] .
\end{aligned}
$$

The function $y$, defined by equations (4) in the interval from $x=0$ to $x=\mathrm{L}$, is therefore represented by the series

$$
\begin{aligned}
\frac{2 \mathrm{~L}}{\pi^{2}} \sum \frac{1}{n^{2}}\left[\frac{\gamma \delta^{\prime}-\gamma^{\prime} \delta}{\gamma\left(\gamma-\gamma^{\prime}\right)}\right. & \sin \frac{n \pi \gamma}{\mathrm{L}} \\
& \left.+\frac{\mathrm{L}\left(\delta-\delta^{\prime}\right)+\gamma \delta^{\prime}-\gamma^{\prime} \delta}{\left(\mathrm{L}-\gamma^{\prime}\right)\left(\gamma-\gamma^{\prime}\right)} \sin \frac{n \pi \gamma^{\prime}}{\mathrm{L}}\right] \sin \frac{n \pi x}{\mathrm{~L}} .
\end{aligned}
$$

The quantities $\gamma, \delta, \gamma^{\prime}, \delta^{\prime}$ must now be so determined as functions of $t$ that this series shall become identical with that defined by (1). This latter is equal to

$$
\frac{b \mathrm{~L}^{2}}{\pi^{2} a(\mathrm{~L}-a)} \Sigma \frac{1}{n^{2}}\left[\sin n \pi\left(\frac{a}{\mathrm{~L}}-\frac{2 t}{\mathrm{~T}}\right)+\sin \pi a\left(\frac{a}{\mathrm{~L}}+\frac{2 t}{\mathrm{~T}}\right)\right] \sin \frac{n \pi x}{\mathrm{~L}} \text {. }
$$

If, therefore, $\mu, \nu$ are undetermined positive or negative whole numbers, we obtain the equations:-

$$
\begin{aligned}
& \left.\begin{array}{l}
\frac{\gamma}{\mathrm{L}}= \pm\left(\frac{a}{\mathrm{~L}}-\frac{2 t}{\mathrm{~T}}+2 \mu\right), \\
\frac{\gamma^{\prime}}{\mathrm{L}}= \pm\left(\frac{a}{\mathrm{~L}}+\frac{2 t}{\mathrm{~T}}+2 v\right) ;
\end{array}\right\} . . . . . . . \\
& \gamma^{\prime}-\gamma^{\prime} \delta= \pm \frac{b \mathrm{~L} \gamma\left(\gamma-\gamma^{\prime}\right)}{2 a(\mathrm{~L}-a)}, \\
& \mathrm{L}\left(\delta-\delta^{\prime}\right)+\gamma \delta^{\prime}-\gamma^{\prime} \delta= \pm \frac{b \mathrm{~L}\left(\mathrm{~L}-\gamma^{\prime}\right)\left(\gamma-\gamma^{\prime}\right)}{2 a(\mathrm{~L}-a)} .
\end{aligned}
$$

The numbers $\mu, \nu$ are to be determined, and their signs chosen, so as to make $\gamma$ and $\gamma^{\prime}$ constantly positive for all values of $t$ between 0 and $\mathrm{T}$, and $\gamma \leqq \gamma^{\prime} \leqq \mathrm{L}$. The same sign must be chosen for the first of equations (5) and the first of equations (6), as well as, on the other hand, for the second equation (5) and the second equation (6). These conditions oblige us to distinguish whether $a>\mathrm{L}-a$ or $a<\mathrm{L}-a$. We assume the former; the other case can be decided in a precisely corresponding manner, and therefore need not be specially treated.

4. First, let $\mu=\nu=0$ and

$$
\frac{\gamma}{\mathrm{L}}=\frac{a}{\mathrm{~L}}-\frac{2 t}{\mathrm{~T}}, \quad \frac{\gamma^{\prime}}{\mathrm{L}}=\frac{a}{\mathrm{~L}}+\frac{2 t}{\mathrm{~T}}
$$


Vilrations of twitched and stroked Strings.

These equations can only exist for

$$
0 \leqq t<\frac{\mathrm{L}-a}{\mathrm{~L}} \cdot \frac{\mathrm{T}}{2} ; . . . . . .
$$

for if $t$ exceeded the given upper limit, $\boldsymbol{\gamma}^{\prime}$ would become $>\mathrm{L}$. From (6) result then

$$
\begin{aligned}
\delta & =\frac{b \boldsymbol{\gamma}}{2 a(\mathrm{~L}-a)}\left(2 \mathrm{~L}-\boldsymbol{\gamma}-\boldsymbol{\gamma}^{\prime}\right)=b \frac{\boldsymbol{\gamma}}{a} ; \\
\delta^{\prime} & =\frac{b\left(\mathrm{~L}-\gamma^{\prime}\right)}{2 a(\mathrm{~L}-a)}\left(\boldsymbol{\gamma}+\boldsymbol{\gamma}^{\prime}\right)=b \frac{\mathrm{L}-\boldsymbol{\gamma}^{\prime}}{\mathrm{L}-\boldsymbol{\gamma}} .
\end{aligned}
$$

Consequently, so long as the inequalities (7) subsist, according to (4) we have:-

$$
\begin{aligned}
& y=b \frac{x}{a} \text { for } 0 \leqq \frac{x}{\mathrm{~L}} \leqq \frac{a}{\mathrm{~L}}-\frac{2 t}{\mathrm{~T}} ; \\
& y=b \frac{\mathrm{L}(a \mathrm{~T}-2 \mathrm{~T} t)+(\mathrm{L}-2 a) x}{2 a(\mathrm{~L}-a) \mathrm{T}} \\
& \text { for } \frac{a}{\mathrm{~L}}-\frac{2 t}{\mathrm{~T}} \leqq \frac{x}{\mathrm{~L}} \leqq \frac{a}{\mathrm{~L}}+\frac{2 t}{\mathrm{~T}} ; \\
& y=b \frac{\mathrm{L}-x}{\mathrm{~L}-a} \text { for } \frac{a}{\mathrm{~L}}+\frac{2 t}{\mathrm{~T}} \leqq \frac{x}{\mathrm{~L}} \leqq 1 . \quad
\end{aligned}
$$

Of the three straight lines, therefore, which make up the figure of the string in the period under consideration, one only changes with the time, and, indeed, that which does not pass through an extremity of the string; it always remains parallel to the straight line

$$
y=\frac{b(\mathrm{~L}-2 a) x}{2 a(\mathrm{~L}-a)} \cdot \text {. . . . . . }
$$

At the close of the time-interval (7), i. e. for

$$
t=\frac{\mathrm{L}-a}{\mathrm{~L}} \cdot \frac{\mathrm{T}}{2}, \quad . \quad . \quad . \quad . \quad \text {. }
$$

$\gamma^{\prime}$ becomes $=\mathrm{L}, \delta^{\prime}=0$; the string now consists of two straight lines, namely

$$
\left.\begin{array}{ll}
y=b \frac{x}{a} & \text { for } 0 \leqq x \leqq 2 a-\mathrm{L}, \\
\gamma=\frac{b(2 a-\mathrm{L})(\mathrm{L}-x)}{2 a(\mathrm{~L}-a)} \quad, 2 a-\mathrm{L} \leqq x \leqq \mathrm{~L} .
\end{array}\right\} .
$$

The second straight line is here again parallel to (9).

5. In the following section of time, defined by

$$
\frac{\mathrm{L}-a}{\mathrm{~L}} \cdot \frac{\mathrm{T}}{2}<t<\frac{a \mathrm{~T}}{2 \mathrm{~L}}, \quad \text {. . . . . }
$$


we have to choose $\mu=0, \nu=-1$, and to put

$$
\frac{\gamma}{\mathrm{L}}=\frac{a}{\mathrm{~L}}-\frac{2 t}{\mathrm{~T}}, \quad \frac{\gamma^{\prime}}{\mathrm{L}}=2-\frac{a}{\mathrm{~L}}-\frac{2 t}{\mathrm{~T}}
$$

Calculating now $\delta$ and $\delta^{\prime}$ from (6)-that is, from

$$
\begin{aligned}
& \gamma \delta^{\prime}-\gamma^{\prime} \delta=\frac{b \mathrm{~L} \gamma\left(\gamma-\gamma^{\prime}\right)}{2 a(\mathrm{~L}-a)}, \\
& \mathrm{L}\left(\delta-\delta^{\prime}\right)+\gamma^{\prime}-\gamma^{\prime} \delta=-\frac{b \mathrm{~L}\left(\mathrm{~L}-\gamma^{\prime}\right)\left(\gamma-\gamma^{\prime}\right)}{2 a(\mathrm{~L}-a)},
\end{aligned}
$$

we get for the interval (12):-

$$
\begin{aligned}
& y=b \frac{x}{a} \text { for } 0 \leqq \frac{x}{\mathrm{~L}} \leqq \frac{a}{\mathrm{~L}}-\frac{2 t}{\mathrm{~T}} ; \\
& y=b \frac{\mathrm{L}(a \mathrm{~T}-2 \mathrm{~L} t)+\mathrm{T}(\mathrm{L}-2 a) x}{2 a(\mathrm{~L}-a) \mathrm{T}} \\
& \text { for } \frac{a}{\mathrm{~L}}-\frac{2 t}{\mathrm{~T}} \leqq \frac{x}{\mathrm{~L}} \leqq 2-\frac{a}{\mathrm{~L}}-\frac{2 t}{\mathrm{~T}} \text {; } \\
& y=-\frac{b}{a}(\mathrm{~L}-x) \text { for } 2-\frac{a}{\mathrm{~L}}-\frac{2 t}{\mathrm{~T}} \leqq \frac{x}{\mathrm{~L}} \leqq 1 \text {. }
\end{aligned}
$$

Again only the middle line depends on $t$; it remains always parallel to (9); the point at which it cuts the $X$ axis now lies between the points $x=0$ and $x=\mathrm{L}$, while it previously lay outside this interval, towards the positive side. The other two segments of the string are parallel to one another, and lie on different sides of the $x$ axis ; the segment adjacent to the point $x=0$ has its direction unchanged.

At the time

$$
t=\frac{a \mathrm{~T}}{2 \mathrm{~L}} \cdot \cdot \cdot \cdot \cdot \cdot \cdot \cdot
$$

the string again consists of only two straight lines, given by

$$
\left.\begin{array}{l}
y=\frac{b(\mathrm{~L}-2 a)}{2 a(\mathrm{~L}-a)} x \text { for } 0 \leqq x \leqq 2(\mathrm{~L}-a), \\
y=-\frac{b}{a}(\mathrm{~L}-x) \quad, \quad 2(\mathrm{~L}-a) \leqq x \leqq \mathrm{~L} .
\end{array}\right\} .
$$

The former of these two lines now coincides with (9). The string lies entirely on the negative side of the $X$ axis.

6. The latter holds also for the entire following time-interval, in which

$$
\frac{a \mathrm{~T}}{2 \mathrm{~L}}<t<\frac{\mathrm{T}}{2}
$$


Again $\mu=0, \nu=-1$; and on the right sides of (5) the negative sign must both times be chosen, so that

$$
\frac{\gamma}{\mathrm{L}}=-\frac{a}{\mathrm{~L}}+\frac{2 t}{\mathrm{~T}}, \quad \frac{\gamma^{\prime}}{\mathrm{L}}=2-\frac{a}{\mathrm{~L}}-\frac{2 t}{\mathrm{~T}} .
$$

Equations (6) therefore become

$$
\begin{aligned}
\gamma \delta^{\prime}-\gamma^{\prime} \delta & =-\frac{b \mathrm{~L} \gamma\left(\gamma-\gamma^{\prime}\right)}{a(\mathrm{~L}-a)}, \\
\mathrm{L}\left(\delta-\delta^{\prime}\right)+\gamma \delta^{\prime}-\gamma^{\prime} \delta & =-\frac{b \mathrm{~L}\left(\mathrm{~L}-\gamma^{\prime}\right)\left(\gamma-\gamma^{\prime}\right)}{a(\mathrm{~L}-a)} .
\end{aligned}
$$

Therefore the string now consists of the three straight lines

$$
\begin{aligned}
& y=-\frac{b}{\mathrm{~L}-a} x \text { for } 0 \leqq \frac{x}{\mathrm{~L}} \leqq \frac{2 t}{\mathrm{~T}}-\frac{a}{\mathrm{~L}} \text {, } \\
& y=b \frac{(a \mathrm{~T}-2 \mathrm{~L} t)+\mathrm{T}(\mathrm{L}-2 a) x}{2 a(\mathrm{~L}-a) \mathrm{T}} \\
& \text { for } \frac{2 t}{\mathrm{~T}}-\frac{a}{\mathrm{~L}} \leqq \frac{x}{\mathrm{~L}} \leqq 2-\frac{a}{\mathrm{~L}}-\frac{2 t}{\mathrm{~T}} \text {, } \\
& y=-\frac{b}{a}(\mathrm{~L}-x) \text { for } 2-\frac{a}{\mathrm{~L}}-\frac{2 t}{\mathrm{~T}} \leqq \frac{x}{\mathrm{~L}} \leqq 1 \text {. }
\end{aligned}
$$

Here also only the middle line is dependent on $t$; it again remains parallel to (9). The straight line passing through $x=0$ is parallel with the third; and that passing through $x=\mathrm{L}$ is parallel with the first line in (8).

At the time $t=\frac{T}{2}, \gamma$ becomes $=\gamma^{\prime}$, and the string consists of the two straight lines

$$
\left.\begin{array}{l}
y=-\frac{b}{\mathrm{~L}-a} x \text { for } 0 \leqq x \leqq \mathrm{~L}-a, \\
y=-\frac{b}{a}(\mathrm{~L}-x), \mathrm{L}-a \leqq x \leqq \mathrm{~L} .
\end{array}\right\} . \cdot \cdot
$$

Consequently it now lies symmetrical with its initial position, as is always the case at the end of half the period of a vibration if the initial velocity of the several points was $=0$. From this point the string returns in a perfectly analogous manner to its initial position : this need not be further enlarged upon. The motion thus presented corresponds with the delineations given by Thomas Young*.

* Conf. loc. cit. suprà, p. 199, and 'The Theory of Sound,' by Lord Rayleigh, vol. i. (London, 1877) p. 185.

By Ch. Delagrave, Libraire-Editeur, a model (first constructed by Monge) is sold, of the surfaces which are generated by the twitched string when it is moved with uniform velocity perpendicularly to its plane. 
Donkin* and Lord Rayleigh enunciated the proposition that the inclination of the string to the position of rest takes two constant values alternately at the two ends. But this is not wholly correct; e.g. for $x=0$ we have, according to the above :-

$$
\begin{aligned}
& \frac{d y}{d x}=\frac{b}{a} \quad \text { for } 0 \leqq t<\frac{a \mathrm{~T}}{2 \mathrm{~L}} ; \\
& \frac{d y}{d x}=\frac{b(\mathrm{~L}-2 a)}{2 a(\mathrm{~L}-a)}, t=\frac{a \mathrm{~T}}{2 \mathrm{~L}} ; \\
& \frac{d y}{d x}=-\frac{b}{\mathrm{~L}-a} \quad \# \frac{a \mathrm{~T}}{2 \mathrm{~L}}<t \leqq \frac{\mathrm{T}}{2} ;
\end{aligned}
$$

\&c. Between each two leaps of the function $\frac{d y}{d x}$ there is therefore a moment when the inclination takes a third value, as at that moment the line (9) itself forms a part of the string. This is in accordance with the circumstance that at a place of discontinuity a trigonometric series represents the mean value.

7. From the foregoing it follows that the series (1) for $0 \leqq t \leqq \frac{\mathrm{T}}{2}$ is constantly a complete linear function of $x$ and $t$, and, indeed, according to the quantity of $t$, like the functions occurring in the equations (8), (11), (13), (15), (16), (17). Therefore series (1) in fact satisfies the differential equation (2), since simultaneously

$$
\frac{\partial^{2} y}{d t^{2}}=0, \quad \frac{\partial^{2} y}{d x^{2}}=0
$$

That the conditions for the possibility of an angle in transversely vibrating strings are fulfilled in the solution by means of a Fourier series was already remarked by Christoffel $l$. $c$. It is easy to verify this in the present case. Here the expressions

$$
\frac{\partial \gamma}{\partial t} \mid \cdot \frac{\partial y}{\partial t}+\alpha^{2} \frac{\partial y}{\partial x}
$$

where $\alpha^{2}$ signifies the quantity oocurring in (2), and

$$
\frac{\partial \gamma}{\partial t} \cdot \frac{\partial y}{\partial x}+\frac{\partial y}{\partial t}
$$

have each the same value on both sides of the angle $\gamma, \delta$. We have to form these expressions for the several time-intervals above distinguished; an easy calculation then shows that they in fact give identical values on both sides of the angle $\gamma, \delta$. The like holds good for the angle $\gamma^{\prime}, \delta^{\prime}$.

* Acoustics, Part I. p. 83: Oxford, 1870. 
Hence the validity of the differential equation (2) is not impaired by the discontinuities of the differential quotients, according to $x$, of series (1), which occur at the angles of the string.

\section{The stroked String.}

8. The notation introduced in no. 2 shall stand also for the violin-string, to be treated of in the following. We base the mathematical treatment of this upon the following proposition, to which direct observations have conducted*, and which represents with approximate accuracy the motion of the several points of the string (conf. infrò, no. 16) :-

Each point in a stroked string vibrates hither and thither with constant velocity; this velocity is different for the different points of the string.

It will be shown that by this the motion of the string is completely determined, if, in addition, the amplitude of any single point of the string is given, and excluding the occurrence of nodes.

We shall find that the velocity of a point of the string (with the exception of the central point) when ascending is necessarily different from the velocity when descending. At first we make no hypothesis about this ; we will only suppose the velocity in each direction separately to be constant.

Let us consider a point $x$ of the string, which at the time $t=0$ commences its ascending motion. The total period of a vibration may be denoted by $\mathrm{T}$, the time of the ascent by $\mathfrak{T}$, therefore that of the descent by $\mathbf{T}-\mathfrak{T}$. We can then put

$$
\left.\begin{array}{lll}
y=a t+h \text { for } \quad 0 \leqq t \leqq \mathfrak{Z}, \\
y=b t=k \quad, \quad \mathfrak{I} \leqq t \leqq \mathrm{~T} .
\end{array}\right\} \quad . \quad .
$$

Since for all values of $t$ the second differential quotient according to $t$ of $y$ is $=0$, this must also be the case with the second differential equation according to $x$ of $y$, by which equation (2) is satisfied; $a, b, h, k$ are consequently complete linear functions of $x$. They are subsequently to be more precisely determined. $\mathfrak{T}$ is also a function of $x$, to be determined, while $\mathrm{T}$ is a constant.

In the determination of these functions the occurrence of node-points shall be excluded.

9. As velocities of opposite motions, $a$ and $b$ are quantities different from one another when they are not both $=0$.

* Conf. Helmholtz l.c. and Clem. Neumann, "Beobachtungen uiber die Schwingungen gestrichener Saiten," Sitzungsberichte der Wiener Akademie 1870, math.-natumo. Classe, Band Ixi. Abth. 2, p. 89.

Phil. Mag. S. 5. Vol. 9. No. 55. March 1880. 
Therefore $\frac{\partial y}{\partial t}$ is discontinuous for $t=\mathfrak{Z}$; that is, at the time $t=\mathfrak{T}$ the point $x$ receives an impulse. Accordingly the formulæ of Christoffel already mentioned in no. 7 become applicable, viz.

$$
\begin{aligned}
& {\left[c \frac{\partial y}{\partial x}+\frac{\partial y}{\partial t}\right]_{+}=\left[c \frac{\partial y}{\partial x}+\frac{\partial y}{\partial t}\right]_{-},} \\
& {\left[c \frac{\partial y}{\partial t}+\alpha^{2} \frac{\partial y}{\partial x}\right]_{+}=\left[c \frac{\partial y}{\partial t}+\alpha^{2} \frac{\partial y}{\partial x}\right]_{-},}
\end{aligned}
$$

in which $\alpha^{2}$ has its previous meaning, and where the two values of the bracketed quantities on the two sides of the place of discontinuity $t=\mathfrak{T}$ are distinguished by the signs + and used as indices. We have

therefore

$$
c=\frac{d x}{d t} \text { for } t=\mathfrak{Z}
$$

$$
\frac{1}{c}=\frac{d \mathfrak{T}}{d x}
$$

Now $a, b$, and $\mathfrak{Z}$ must be continuous functions of $x$, unless we suppose the connexion of the string broken. In like manner $h$ and $k$ are continuous ; for otherwise $\frac{\partial y}{\partial x}$, and consequently also $\frac{\partial y}{\partial t}$, would be discontinuous at other times as well. Even $\frac{d \mathfrak{Z}}{d x}$ is a continuous function of $x$; for since all the points will not simultaneously be at the greatest distance from the position of equilibrium, for a variable point $x$ equations (18) are to be replaced by

$$
\left.\begin{array}{l}
y=a t+h \text { for } \mathfrak{I}_{0} \leqq t \leqq \mathfrak{T}_{0}+\mathfrak{T}, \\
y=b t+k \quad, \quad \mathfrak{I}_{0}+\mathfrak{T} \leqq t \leqq \mathfrak{I}_{0}+\mathrm{T},
\end{array}\right\} \quad \cdot \quad \cdot
$$

when the point $x$ arrives at its extreme position at the time $t=\mathfrak{T}_{0}$, where $\mathfrak{T}_{0}$ is a continuous function of $x$. As the motion is to have the period $\mathrm{T}$,

$$
a \mathfrak{Z}_{0}+h=b\left(\mathfrak{T}_{0}+\mathrm{T}\right)+k \text {. }
$$

This holds good for every point $x$ of the string. Forming the corresponding equation for $x+d x$ and for $x-d x$, and distinguishing the differentials taken in the positive and negative directions by the indices + and - , there follows, by subtraction of the two resulting equations:-

$$
a\left[\left(\frac{d \mathfrak{T}_{0}}{d x}\right)_{+}-\left(\frac{d \mathfrak{I}_{0}}{d x}\right)_{-}\right]=b\left[\left(\frac{d \mathfrak{I}_{0}}{d x}\right)_{+}-\left(\frac{d \mathfrak{I}_{0}}{d x}\right)_{-}\right] .
$$


Therefore either $a=b$, or $\frac{d \boldsymbol{\Sigma}_{0}}{d x}$ is continuous.

Further, in order to preserve the continuity of the string, the condition

$$
a\left(\mathfrak{Z}_{0}+\mathfrak{Z}\right)+h=b\left(\mathfrak{I}_{0}+\mathfrak{T}\right)+k
$$

must be fulfilled. From this, in an analogous manner, it follows that $\frac{d\left(\mathfrak{T}_{0}+\mathfrak{T}\right)}{d x}$, and consequently also $\frac{\delta \mathfrak{T}}{d x}$, is continuous as soon as $a$ is different from $b$. But if we assume that $a=b$, the last condition gives $h=k$, and the one before made use of gives $h=a \mathrm{~T}+k$; therefore must $a=b=0$, as we have already remarked (at the commencement of this no.). Hence $\frac{d \mathfrak{Z}}{d x}$ can become discontinuious only at a node-point* ; but we have excluded the occurrence of nodes. By this the above assertion is proved. It was necessary to use this demonstration, since in Christoffel's investigations the continuous progression of the place of discontinuity is presupposed.

In consequence of these discussions the above conditions of discontinuity can be applied, and, taking $\left(18^{*}\right)$ into consideration, can be written in the following form:-

$$
\begin{aligned}
& \left(\frac{\partial y}{\partial x}\right)_{+}+a \frac{d\left(\mathfrak{I}_{0}+\mathfrak{T}\right)}{d x}=\left(\frac{\partial y}{\partial x}\right)_{-}+b \frac{d\left(\mathfrak{I}_{0}+\mathfrak{T}\right)}{d x} ; . \\
& a+\alpha^{2}\left(\frac{\partial y}{\partial x}\right)_{+} \frac{d\left(\mathfrak{I}_{0}=\mathfrak{I}\right)}{d x}=b+\alpha^{2}\left(\frac{\partial y}{\partial x}\right)_{-} \frac{d\left(\mathfrak{I}_{0}+\mathfrak{T}\right)}{d x} .
\end{aligned}
$$

Hence it follows that at no place in the string can $\frac{d\left(\mathfrak{Z}_{0}+\mathfrak{Z}\right)}{d x}$ vanish; for otherwise $a$ would necessarily be $=b$, which is impossible (conf. suprà). Therefore

The function $\mathfrak{Z}_{0}+\mathfrak{T}$ has in the interval from $x=0$ to $x=\mathrm{L}$ neither a maximum nor a minimum; it increases continuously, or it decreases continuously.

10. Hence in the interval mentioned there is only one value of $x$ which satisfies the equation $t=\mathfrak{Z}_{0}+\mathfrak{Z}$. That is, at any time $t$ the string has only one angle. Or, since $y$, according to no. 8 , is a complete linear function of $x$,

At any time whatever the string consists of two straight lines.

From this immediately follows :-

All the points of the string pass simultaneously through the position of equilibrium.

On account of the latter circumstance it is advisable to select as the point of time $t=0$ that in which all the points $x$

* In a node such discontinuities actually occur: conf: infra, no, 15. 
pass through the position of equilibrium; for the moment $t=0$ selected in equations (18) appears essentially dependent on $x$. In consequence of this new determination equations (18) change into :-

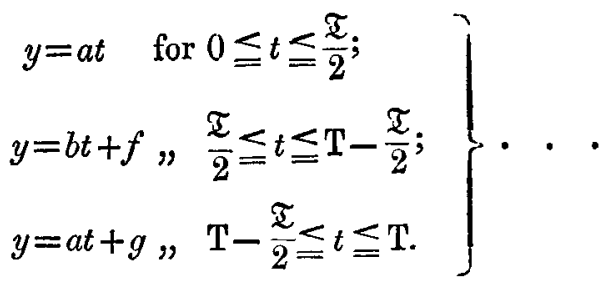

$a, b, f, g$ are complete linear functions, to be determined, of $x$. Between them and the function $\mathfrak{I}$ a series of relations subsist.

At the time T', namely, every point must again be in the position of equilibrium ; therefore

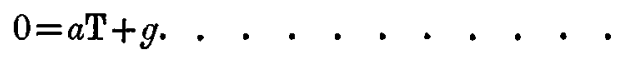

Further, we have

and thence

$$
\begin{aligned}
& a \mathfrak{T}=b \mathfrak{T}+2 f, \quad \cdot \quad \cdot \quad \cdot \quad \cdot \quad \cdot \cdot \\
& b(2 \mathrm{~T}-\mathfrak{T})+2 f=a(2 \mathrm{~T}-\mathfrak{T})+2 g, \quad . \quad .
\end{aligned}
$$

$$
a \mathrm{~T}=-b(\mathrm{~T}-\mathfrak{T}) . \cdot \cdot \cdot \cdot \cdot \cdot \cdot \cdot
$$

This last equation affirms that the path travelled in ascending differs from that travelled in descending only in its sign, as must be the case in a periodic vibration.

11. Respecting $\mathfrak{I}$, according to no. 9 two different assumptions can bo made: either $\mathfrak{T}$ increases when $x$ increases; or $\mathfrak{I}$ diminishes as $x$ increases. In both cases for $x=0$ and $x=\mathrm{L}$, i. e. for the extremities of the string, must

$$
\mathfrak{T} \equiv 0 \text { mod. } \mathrm{T} \text {. }
$$

In the former case, therefore, we can take $\mathfrak{I}=0$ for $x=0$, and consequently (since no nodes are supposed to be present, and since $\mathfrak{T}$ increases simultaneously with $x$ increasing) $\mathfrak{T}=\mathrm{T}$ for $x=\mathrm{L}$; that is, the points in the vicinity of $x=0$ rise very quickly and descend very slowly, while the neighbouring points to $x=\mathrm{L}$ descend rapidly and ascend very slowly. In the other case the behavionr of the two ends of the string is exactly the reverse. In the following we make the former assumption; the other would be decided simply by the exchange of $\mathrm{x}$ with $\mathrm{L}-\mathrm{x}$. 
According to this, $x$ can always be chosen so small that, for a given value of $t$,

$$
\overline{\mathfrak{T}} \leqq t \leqq \mathrm{~T}-\frac{\mathfrak{I}}{2}
$$

For points of the string which are situated sufficiently near the end $x=0$, therefore, the middle equation of (21) always holds good; so that for them $y=b t+f$. For $x=0$ must $y=0$; consequently we can put

$$
b=\mathrm{B} x, f=\mathrm{F} x,
$$

where $B$ and $F$ denote constants.

The function $\mathfrak{T}$ reaches its greatest value for $x=\mathrm{L}$. Therefore, if $2 t<\mathrm{T}$, then the first of the equations (21) holds good in the vicinity of the end $x=\mathrm{L}$; it must give $y=0$ for $x=\mathrm{L}$; we thus get

$$
a=\mathrm{A}(\mathrm{L}-x),
$$

where A denotes a constant.

It then follows, further, from (22), that

$$
g=-\mathrm{AT}(\mathrm{L}-x) \text {. }
$$

In fact, $g$ must contain the factor $\mathrm{L}-x$, since for $x=\mathrm{L}$ and $2 t>\mathrm{T}$ the third of the equations (21) is valid.

The function $\mathfrak{I}$ is now determined from (25); we get

$$
\mathfrak{I}=\frac{-b \mathrm{~T}}{a-b}=\frac{-\mathrm{BT} x}{\mathrm{~A}(\mathrm{~L}-x)-\mathrm{B} x} ;
$$

lastly, $f$ is found from (23)-namely,

$$
f=\mathrm{F} x=\frac{\mathfrak{T}}{2}(a-b)=-\frac{1}{2} \mathrm{BT} x .
$$

12. The constants $A$ and $B$, by which all is expressed, can be reduced to a single constant by means of the discontinuityconditions (19) and (20). In correspondence with the replacing of equations (18) by (21), $\mathfrak{I}$ must previously be replaced in these relations by $\frac{1}{2} \mathfrak{T}$ or by $\mathrm{T}-\frac{1}{2} \mathfrak{T}$.

For the place of discontinuity $t=\frac{1}{2} \mathfrak{I}$ both sides of equation (19) become, by virtue of the values just found for $a, b, f, g$, and $\mathfrak{T}$, equal to

$$
-\mathrm{ABT} \frac{\mathrm{A}(\mathrm{L}-x)^{2}+\mathrm{B} x^{2}}{2[\mathrm{~A}(\mathrm{~L}-x)-\mathrm{B} x]^{2}}
$$

This relation is therefore spontaneously fulfilled.

Equation (20), on the contrary, gives for the same place of discontinuity:- 


$$
\begin{gathered}
4 \mathrm{~A}(\mathrm{~A}+\mathrm{B})^{3} x^{4}-4 \mathrm{AL}(\mathrm{A}+\mathrm{B})^{2}(4 \mathrm{~A}+\mathrm{B}) x^{3} \\
+12 \mathrm{~A}^{2} \mathrm{~L}^{2}(\mathrm{~A}+\mathrm{B})(2 \mathrm{~A}+\mathrm{B}) x^{2} \\
-\mathrm{A}^{2} \mathrm{~L}\left[12 \mathrm{AL}^{2}(\mathrm{~A}+\mathrm{B})+4 \mathrm{~A}^{2} \mathrm{~L}^{2}+\alpha^{2} \mathrm{~B}^{2} \mathrm{~T}^{2}\right] x+4 \mathrm{~A}^{4} \mathrm{~L}^{4} \\
=-4 \mathrm{~B}(\mathrm{~A}+\mathrm{B})^{3} x^{4}+12 \mathrm{ABL}(\mathrm{A}+\mathrm{B})^{2} x^{3} \\
-12 \mathrm{~A}^{2} \mathrm{BL} \mathrm{L}^{2}(\mathrm{~A}+\mathrm{B}) x^{2}+\mathrm{A}^{2} \mathrm{BL}\left(4 \mathrm{AL} \mathrm{L}^{2}-\alpha^{2} \mathrm{BT}^{2}\right) x \\
+\alpha^{2} \mathrm{~A}^{2} \mathrm{~B}^{2} \mathrm{~L}^{2} \mathrm{~T}^{2} .
\end{gathered}
$$

This equation has to subsist for all values of $x$, which can only be the case if

$$
\mathrm{A}+\mathrm{B}=0 \text { and } \alpha^{2}=\frac{4 \mathrm{~A}^{2} \mathrm{~L}^{2}}{\mathrm{~B}^{2} \mathrm{~T}^{2}}=\frac{4 \mathrm{~L}^{2}}{\mathrm{~T}^{2}} .
$$

The second of these conditions shows us the dependence between the vibration-period, on the one hand, and the length, mass, and tension, of the string, on the other (conf. no. 2); it is the same relation which is otherwise obtained from the theory of Fourrier's series for transversal vibrations. Here it was to be demonstrated in another way; the same could also have been done in the well-known manner with the aid of d'Alembert's solution.

The first of the relations found expresses $B$ in terms of $A$. The functions $a, b, f, g, \mathfrak{T}$, determined in no. 11, now become

$$
\begin{aligned}
& b=-\mathrm{A} x, \quad f=\frac{1}{2} \mathrm{AT} x, \\
& a=\mathrm{A}(\mathrm{L}-x), \quad g=\mathrm{AT}\left(\mathrm{L}_{-}-x\right), \\
& \mathfrak{I}=\frac{\mathrm{T}}{\mathrm{L}} x^{*} . . .
\end{aligned}
$$

Herewith the relations subsisting for the discontinuity-place $t=\mathrm{T}-\frac{1}{2} \mathfrak{T}$ are spontaneously fulfilled.

Accordingly equations (21) change into

$$
\left.\begin{array}{ll}
y=\mathrm{A}(\mathrm{L}-x) t & \text { for } 0 \leqq \frac{t}{\mathrm{~T}} \leqq \frac{x}{2 \mathrm{~L}}, \\
y=\mathrm{A} x\left(\frac{\mathrm{T}}{2}-t\right) & , \frac{x}{2 \mathrm{~L}} \leqq \frac{t}{\mathrm{~T}} \leqq \frac{2 \mathrm{~L}-x}{2 \mathrm{~L}}, \\
y=-\mathrm{A}(\mathrm{L}-x)(\mathrm{T}-t) & , \frac{2 \mathrm{~L}-x}{2 \mathrm{~L}} \leqq \frac{t}{\mathrm{~T}} \leqq 1 .
\end{array}\right\} .
$$

By these the motion of the string is represented completely.

* In the determination of $\mathfrak{T}$ we might have started from the relation $a^{2}\left(\frac{1}{2} \frac{d S_{2}}{d x}\right)^{2}=1$, which, according to Christoffel, must be fulfilled, and by virtue of which one of the conditions of discontinuity results from the other. 
The constant $\mathrm{A}$ is determined by the amplitude of any point of the string which is equal to $\frac{\mathrm{AT}}{2 \mathrm{~L}}(\mathrm{~L}-x) x$. Therefore, if the amplitude of the central point of the string is denoted by $P$, we have $\mathrm{P}=\frac{\mathrm{ALT}}{8}$, and consequently

$$
\mathrm{A}=\frac{8 \mathrm{P}}{\mathrm{LT}^{*}} \quad \cdot \quad \cdot \quad \cdot . .
$$

According to. Neumann's experiments (conf. no. 8) P depends on the place at which the string is stroked, and on the velocity with which the bow is drawn across; that is to say, the velocity of the stroked point is, in ascending, equal to that of the bow. Let $\mathrm{V}$ be this velocity, and $a=\lambda$ the strokingplace; then, according to (27) and (28),

$$
\mathrm{V}=\mathrm{A}(\mathrm{L}-\lambda), \mathrm{P}=\frac{\mathrm{VLT}}{8(\mathrm{~L}-\lambda)} .
$$

This, however, holds good only when the stroking-place lies pretty near one end of the string (conf. the end of no. 1).

13. In detail, the motion of the string takes place in the following manner :-

At the time $t=0$ it is in the position of equilibrium.

If $0<t<\frac{T}{2}$, there is one point of the string for which $\mathrm{T}-\frac{x \mathrm{~T}}{2 \mathrm{~L}}$ would be $<t$; hence it consists of the two straight lines

$$
y=\mathrm{A}(\mathrm{L}-x) t, y=\mathrm{A} x\left(\frac{\mathrm{T}}{2}-t\right) .
$$

To their point of intersection corresponds the abscissa

$$
x=2 \mathrm{~L} \frac{t}{\mathrm{~T}} \text {. }
$$

It moves upon the parabola

$$
y=\frac{\mathrm{AT}}{2 \mathrm{~L}}(\mathrm{~L}-x) x . . . \quad . \quad . \quad .
$$

from $x=0$ to $x=\mathrm{L}$ while $t$ increases from 0 to $\frac{\mathrm{T}}{2}$.

For $t=\frac{T}{2}$, only the middle equation of (27) holds good; we have therefore $y=0$; that is, at the expiration of the half vibration-period all the points of the string pass through the position of equilibrium.

For $t>\frac{T}{2}$ the two equations 


$$
y=\mathrm{A} x\left(\frac{\mathrm{T}}{2}-t\right), \quad y=-\mathrm{A}(\mathrm{L}-x)(\mathrm{T}-t)
$$

hold good.

The string is deflected from the position of equilibrium in the opposite direction, $y$ beooming negative, while previously (when $\mathrm{A}>0$ ) it was positive. It consists of two straight lines whose point of intersection passes along the parabola symmetrical to (29)

$$
y=-\frac{\mathrm{AT}}{2 \mathrm{~L}}(\mathrm{~L}=x) x, . . . .
$$

in the direction from $x=\mathrm{L}$ to $x=0$.

Lastly, for $t=\mathrm{T}$ the last of the equations (27) subsists; the string has returned to its initial position.

The parabolas (29) and (30) would be travelled in the opposite direction by the angle of the string on the second of the two suppositions respecting $\mathfrak{I}$ possible according to no. 11 and, in accordance therewith, the substitution of $\mathrm{L}-x$ for $x$ in the foregoing formula.

14. It is of importance for acoustics that the function $y$, defined by (27), be expanded into a Fourrier series. Since $y=0$ for $t=0$ and $t=\frac{T}{2}$, a series of sines will be chosen so that

$$
\begin{aligned}
& y=\Sigma \mathrm{B}_{n} \sin \frac{2 n \pi t}{\mathrm{~T}}, \\
& \mathrm{~B}_{n}=\frac{2}{\mathrm{~T}} \int_{0}^{\mathrm{T}} y \sin \frac{2 n \pi t}{\mathrm{~T}} d t=\frac{\mathrm{ALT}}{n^{2} \pi^{2}} \sin \frac{n \pi a}{\mathrm{~L}} .
\end{aligned}
$$

If, in addition, by means of (28) the amplitude $\mathrm{P}$ of the central point of the string be introduced, we shall get for $y$ the following expansion into a trigonometrical series :-

$$
y=\frac{8 \mathrm{P}}{n^{2}} \Sigma \frac{1}{n^{2}} \sin \frac{n \pi x}{\mathrm{~L}} \sin \frac{2 n \pi t}{\mathrm{~T}} . . . . .
$$

This is of the same form as the series which otherwise occur with vibrating strings.

According to formula (31), besides the fundamental tone all the upper tones are contained in the sound of the string. But if the stroking-place happens to be at a point marking an aliquot part, those upper tones are wanting (according to the observations of Helmholtz and Neumann) which have a nodepoint at the stroking-place. This fact does not obtain expression in formula (31). Honce, although the above-described motion of the violin-string agrees very well with Neumann's stroboscopic observations, the hypothesis enunciated in no. 8 
can only be designated as approximately accordant. On this account we will now give another treatment of the motion of the violin-string, more closely agreeing with the facts, for the case that the bow acts at a node-point of an upper tone.

15. For our purpose it is expedient at first to let the supposition made in no. 8 stand, but now to take into account the occurrence of node-points, which has hitherto been excluded.

The occurrence of node-points is usually settled with the aid of Fourrier's series. This, however, is not necessary ; for d'Alembert's solution conducts to similar results. The latter can be applied in our case also ; we can therefore assume as known that the points

$$
x=\frac{2}{m} \mathrm{~L}, \quad x=\frac{3}{m} \mathrm{~L}, \ldots x=\frac{m-1}{m} \mathrm{~L}
$$

must be node-points as soon as the point $x=\frac{\mathrm{L}}{m}$ is a node, and that every portion of the string vibrates separately as an independent string, each two adjacent parts vibrating symmetrically to one another. If, then, $A$ is a constant and $T$ the vibration-period of a division of the string, we have, according to (27),

$$
\begin{gathered}
\text { for } 0 \leqq x \leqq \frac{\mathrm{L}}{m}:- \\
y=\mathrm{A}\left(\frac{\mathrm{L}}{m}-x\right) t \quad \text { for } \quad 0 \leqq \frac{t}{\mathrm{~T}} \leqq \frac{m x}{2 \mathrm{~L}}, \\
y=\mathrm{A} x\left(\frac{\mathrm{T}}{2}-t\right) \quad, \quad \frac{m x}{2 \mathrm{~L}} \leqq \frac{t}{\mathrm{~T}} \leqq \frac{2 \mathrm{~L}-m x}{2 \mathrm{~L}}, \\
y=-\mathrm{A}\left(\frac{\mathrm{L}}{m}-x\right)(\mathrm{T}-t)
\end{gathered}
$$

where now $\frac{2 \mathrm{~L}}{m \mathrm{~T}}=\alpha$, if $\alpha$ denotes the fundamental constant of the differential equation (2).

Further, for $\frac{\mathrm{L}}{m} \leqq x \leqq \frac{\mathrm{L}}{m}$ :-

$$
\begin{aligned}
& y=\mathrm{A}\left(\frac{\mathrm{L}}{m}-x\right) t \quad \text { for } 0 \leqq \frac{t}{\mathrm{~T}} \leqq \frac{2 \mathrm{~L}-m x}{2 \mathrm{~L}} ; \\
& y=-\mathrm{A}\left(\frac{2 \mathrm{~L}}{m}-x\right)\left(\frac{\mathrm{T}}{2}-t\right), \quad \frac{2 \mathrm{~L}-m x}{2 \mathrm{~L}} \leqq \frac{t}{\mathrm{~T}} \leqq \frac{m x}{2 \mathrm{~L}} \\
& y=-\mathrm{A}\left(\frac{\mathrm{L}}{m}-x\right)(\mathrm{T}-t) \quad, \frac{m x}{2 \mathrm{~L} \leqq} \leqq \frac{t}{\mathrm{~T}} \leqq 1
\end{aligned}
$$


In the next interval, where $\frac{2 \mathrm{~L}}{m} \leqq x \leqq \frac{3 \mathrm{~L}}{m}$, we should have:-

$$
\begin{aligned}
& y=\mathrm{A}\left(\frac{3 \mathrm{~L}}{m}-x\right) t \quad \text { for } 0 \leq \frac{t}{\mathrm{~T}} \leqq \frac{m x-2 \mathrm{~L}}{2 \mathrm{~L}} ; \\
& y=\mathrm{A}\left(x-\frac{2 \mathrm{~L}}{m}\right)\left(\frac{\mathrm{T}}{2}-t\right) t, \frac{m x-2 \mathrm{~L}}{2 \mathrm{~L}} \leqq \frac{t}{\mathrm{~T}} \leqq \frac{4 \mathrm{~L}-m x}{2 \mathrm{~L}} ; \\
& y=-\mathrm{A}\left(\frac{3 \mathrm{~L}}{m}-x\right)(\mathrm{T}-t), \frac{4 \mathrm{~L}-m x}{2 \mathrm{~L}} \leqq \frac{t}{\mathrm{~T}} \leqq 1 .
\end{aligned}
$$

Lastly, we find generally, when

$$
\frac{2 i}{m} \mathrm{~L} \leqq x \leqq \frac{2 i+1}{m} \mathrm{~L}:-
$$

$$
\begin{aligned}
& y=\mathrm{A}\left(\frac{2 i+1}{m} \mathrm{~L}-x\right) t \quad \text { for } 0 \leqq \frac{t}{\mathrm{~T}} \leqq \frac{m x-2 i \mathrm{~L}}{2 \mathrm{~L}} ; \\
& y=\mathrm{A}\left(x-\frac{2 i}{m} \mathrm{~L}\right)\left(\frac{\mathrm{T}}{2}-t\right), \frac{m x-2 i \mathrm{~L}}{2 \mathrm{~L}} \leqq \frac{t}{\mathrm{~T}} \leqq \frac{2(i+1) \mathrm{L}-m x}{2 \mathrm{~L}} ; \\
& y=-\mathrm{A}\left(\frac{2 i+1}{m} \mathrm{~L}-x\right)(\mathrm{T}-t) \text { for } \frac{2(i+1) \mathrm{L}-m x}{2 \mathrm{~L}} \leqq \frac{t}{\mathrm{~T}} \leq 1 .
\end{aligned}
$$

And when $\frac{2 i-1}{m} \mathrm{~L} \leqq x \leqq \frac{2 i}{m} \mathrm{~L}:-$

$$
\begin{aligned}
& y=\mathrm{A}\left(\frac{2 i-1}{m} \mathrm{~L}-x\right) t \quad \text { for } 0 \leqq \frac{t}{\mathrm{~T}} \leqq \frac{2 i \mathrm{~L}-m x}{2 \mathrm{~L}} ; \\
& y=-\mathrm{A}\left(\frac{2 i}{m} \mathrm{~L}-x\right)\left(\frac{\mathrm{T}}{2}-t\right), \frac{2 i \mathrm{~L}-m x}{2 \mathrm{~L}} \leqq \frac{t}{\mathrm{~T}} \leqq \frac{m x-2(i-1) \mathrm{L}}{2 \mathrm{~L}} ; \\
& y=-\mathrm{A}\left(\frac{2 i-1}{m} \mathrm{~L}-x\right)(\mathrm{T}-t) \text { for } \frac{m x-2(i-1) \mathrm{L}}{2 \mathrm{~L}} \leqq \frac{t}{\mathrm{~T}} \leqq 1 .
\end{aligned}
$$

Since $y$ is a function $\phi(x)$ of $x$ satisfying the conditions

$$
\begin{aligned}
& \phi\left(x+\frac{2 \mathrm{~L}}{m}\right)=\phi(x), \\
& \phi\left(x+\frac{\mathrm{L}}{m}\right)=-\phi\left(\frac{2 \mathrm{~L}}{m}-x\right),
\end{aligned}
$$

in expanding it into a series of sines we need only make use of equations (32). We find (analogously to no. 14)

$$
y=\frac{\operatorname{ALT}}{m \pi^{2}} \Sigma \frac{1}{n^{2}} \sin \frac{n m \pi x}{\mathrm{~L}} \sin \frac{2 n \pi t}{\mathrm{~T}} \text {. }
$$

16. It shall now be assumed, further, that the several portions of the string vibrate by themselves, as in the preceding 
Vibrations of twitched and stroked Strings.

case, but that the points

$$
x=\frac{\mathrm{L}}{m}, \quad x=\frac{2 \mathrm{~L}}{m}, \ldots x=\frac{m-1}{m} \mathrm{~L},
$$

which just now as node-points remained fixed, themselves execute vibrations in accordance with the law enunciated in (27). Evidently we have then

$$
y=\eta+\Delta \eta
$$

if $\eta$ signifies the function denoted in (27) by $y$, and if $\Delta \eta$ signifies the function denoted in no. 15 by $y$; only we must in the latter substitute for $A$ another constant $B$, and replace $T$ by $\frac{\mathrm{T}}{m}$.

We thus find, if $0 \leqq x \leqq \frac{\mathrm{L}}{m}$,

$$
\left.\begin{array}{c}
y=\left[\mathrm{A}(\mathrm{L}-x)+\mathrm{B}\left(\frac{\mathrm{L}}{m}-x\right)\right] t \quad \text { for } 0 \leqq \frac{t}{=} \leqq \begin{array}{c}
\mathrm{T} \\
2 \mathrm{~L}
\end{array} \\
y=\left[\mathrm{A}\left(\frac{\mathrm{T}}{2}-t\right)+\mathrm{B}\left(\frac{\mathrm{T}}{2 m}-t\right)\right] x, \quad, \frac{x}{2 \mathrm{~L}} \leqq \frac{t}{\mathrm{~T}} \leqq \frac{2 \mathrm{~L}-m x}{2 m \mathrm{~L}}, \\
y=\mathrm{A} x\left(\frac{\mathrm{T}}{2}-t\right)-\mathrm{B}\left(\frac{\mathrm{L}}{m}-x\right)\left(\frac{\mathrm{T}}{m}-t\right) \\
\text { for } \frac{2 \mathrm{~L}-m x}{2 m \mathrm{~L}} \leqq \frac{t}{\mathrm{~T}} \leqq \frac{2 \mathrm{~L}+m x}{2 m \mathrm{~L}}, \\
y=\left[\mathrm{A}\left(\frac{\mathrm{T}}{2}-t\right)+\mathrm{B}\left(\frac{3 \mathrm{~T}}{2 m}-t\right)\right]^{\prime} x \\
\text { for } \frac{2 \mathrm{~L}+m x}{2 m \mathrm{~L}} \leqq \frac{t}{\mathrm{~T}} \leqq \frac{4 \mathrm{~L}-m x}{2 m \mathrm{~L}}, \& \mathrm{c} .
\end{array}\right\}
$$

We have generally:-

$$
\begin{gathered}
y=\left[\mathrm{A}\left(\frac{\mathrm{T}}{2}-t\right)+\mathrm{B}\left(\frac{2 i-1}{2 m} \mathrm{~T}-t\right)\right] x \\
\text { for } \frac{2(i-1) \mathrm{L}-m x}{2 m \mathrm{~L}} \leqq \frac{t}{\mathrm{~T}} \leqq \frac{2 i \mathrm{~L}-m x}{2 m \mathrm{~L}} ; \\
y=\mathrm{A} x\left(\frac{\mathrm{T}}{2}-t\right)-\mathrm{B}\left(\frac{\mathrm{L}}{m}-x\right)\left(\frac{i \mathrm{~T}}{m}-t\right) \\
\text { for } \frac{2 i \mathrm{~L}-m x}{2 m \mathrm{~L}} \leqq \frac{t}{\mathrm{~T}} \leq \frac{2 i \mathrm{~L}+m x}{2 m \mathrm{~L}} ; \\
\left.y=\left[\mathrm{A} \frac{\mathrm{T}}{2}-t\right)+\mathrm{B}\left(\frac{2 i+1}{2 m} \mathrm{~T}-t\right)\right] x \\
\text { for } \frac{2 i \mathrm{~L}+m x}{2 m \mathrm{~L}} \leqq \frac{t}{\mathrm{~T}} \leqq \frac{2(i+1) \mathrm{L}-m x}{2 m \mathrm{~L}} .
\end{gathered}
$$


This system of three equations is to be formed successively for $i=1,3,5, \ldots m-1$ when $m$ is even, for $i=1,3,5, \ldots m-2$ when $m$ is odd.

In the former case the operation finishes with the last of these three equations, formed for $i=m-1$; in the other case, with the first, formed for $i=m$. Therefore, when $m$ is even, the last equation reads

$$
\begin{aligned}
& y=\left[\mathrm{A}\left(\frac{\mathrm{T}}{2}-t\right)+\mathrm{B}\left(\frac{2 m-1}{2 m} \mathrm{~T}-t\right)\right] x \\
& \quad \text { for } \frac{2(m-1) \mathrm{L}+m a}{2 n \mathrm{~L}} \leqq \frac{t}{\mathrm{~T}} \leqq \frac{2 \mathrm{~L}-x}{2 \mathrm{~L}} ;
\end{aligned}
$$

and when $m$ is odd,

$$
\begin{aligned}
y=\left[\mathrm{A}\left(\frac{\mathrm{T}}{2}-t\right)+\mathrm{B}\left(\frac{2 m-1}{2 m} \mathrm{~T}-t\right)\right] x & \\
& \text { for } \frac{2(m-1) \mathrm{L}-m x}{2 m \mathrm{~L}} \leqq \frac{t}{\mathrm{~T}} \leqq \frac{2 \mathrm{~L}-x}{2 \mathrm{~L}} .
\end{aligned}
$$

The equation

$$
\begin{aligned}
& y=-\mathrm{A}(\mathrm{L}-x)(\mathrm{T}-t)-\mathrm{B}\left(\frac{\mathrm{L}}{m}-x\right)\left(\frac{\mathrm{T}}{m}-t\right) \\
& \qquad \text { for } \frac{2 \mathrm{~L}-x}{2 \mathrm{~L}} \leqq \frac{t}{\mathrm{~T}} \leqq 1 .
\end{aligned}
$$

must be superadded, in order to have the motion in the interval of time from $t=0$ to $t=\mathrm{T}$ represented for all the points between $x=0$ and $x=\frac{\mathrm{L}}{\mathrm{m}}$. From (35) and (36) it follows that the ascending motion, for the part of the string considered, proceeds with the constant velocity

$$
\mathrm{A}(\mathrm{L}-x)+\mathrm{B}\left(\frac{\mathrm{L}}{m}-x\right)
$$

while the velocity of the descending motion fluctuates between the values

$$
-(\mathrm{A}+\mathrm{B}) x, \quad-\mathrm{A} x+\mathrm{B}\left(\frac{\mathrm{L}}{m}-x\right), \quad-(\mathrm{A}+\mathrm{B}) x, \ldots
$$

17. The general formula can be constructed in an analogous manner. We have, when

$$
\begin{gathered}
\frac{2 i}{m} \mathrm{~L} \leqq x \leqq \frac{2 i+1}{m} \mathrm{~L}:- \\
y=\mathrm{A}(\mathrm{L}-x) t+\mathrm{B}\left(\frac{2 i+1}{m} \mathrm{~L}-x\right)\left(t-\frac{j-1}{m} \mathrm{~T}\right) \\
\quad \text { for } \frac{j-1}{m} \leqq \frac{t}{\mathrm{~T}} \leqq \frac{m x-2(i-j+1) \mathrm{L}}{2 m \mathrm{~L}}
\end{gathered}
$$


Vibrations of twitched and stroked Strings.

$$
\begin{aligned}
& y=\mathrm{A}(\mathrm{L}-x) t+\mathrm{B}\left(x-\frac{2 i}{m} \mathrm{~L}\right)\left(\frac{j+1}{2 m} \mathrm{~T}-t\right) \\
& \text { for } \frac{m x-2(i-j+1) \mathrm{L}}{2 m \mathrm{~L}} \leqq \frac{t}{\mathrm{~T}} \leqq \frac{2(i+j) \mathrm{L}-m x}{2 m \mathrm{~L}} ; \\
& y=\mathrm{A}(\mathrm{L}-x) t-\mathrm{B}\left(\frac{2 i+1}{m} \mathrm{~L}-x\right)\left(\frac{j}{m} \mathrm{~T}-t\right) \\
& \text { for } \frac{2(i+j) \mathrm{L}-m x}{2 m \mathrm{~L}} \leqq \frac{t}{\mathrm{~T}} \leqq \frac{j}{m} .
\end{aligned}
$$

These equations are to be formed for $j=1,2,3, \ldots i$; and then the first of them, formed for $j=i+1$, is to be superadded -namely,

$$
\begin{aligned}
& y=\mathrm{A}(\mathrm{L}+x) t+\mathrm{B}\left(\frac{2 i+1}{m} \mathrm{~L}-x\right)\left(t-\frac{i}{m} \mathrm{~T}\right) \\
& \qquad \text { for } \frac{i}{m} \leqq \frac{t}{\mathrm{~T}} \leqq \frac{x}{2 \mathrm{~L}} .
\end{aligned}
$$

Thereby the first half of the ascending motion is determined; its velocity fluctuates between the values

and

$$
\mathrm{A}(\mathrm{L}-x)+\mathrm{B}\left(\frac{2 i+1}{m} \mathrm{~L}-x\right)
$$

$$
\mathrm{A}(\mathrm{L}-x)+\mathrm{B}\left(\frac{2 i}{m} \mathrm{~L}-x\right) .
$$

For the descending motion we find:-

$$
\begin{aligned}
& y=\mathrm{A} x\left(\frac{\mathrm{T}}{2}-t\right)+\mathrm{B}\left(x-\frac{2 i}{m} \mathrm{~L}\right)\left(\frac{i+j+1}{m} \mathrm{~T}-t\right) \\
& \text { for } \frac{m x+2(j-1) \mathrm{L}}{2 m \mathrm{~L}} \leqq \frac{t}{\mathrm{~T}} \leqq \frac{2(2 i+j) \mathrm{L}-m x}{2 m \mathrm{~L}} ; \\
& y=\mathrm{A} x\left(\frac{\mathrm{T}}{2}-t\right)-\mathrm{B}\left(\frac{2 i+1}{m} \mathrm{~L}-x\right)\left(\frac{i+j}{m} \mathrm{~T}-t\right) \\
& \text { for } \frac{2(2 i+j) \mathrm{L}-m x}{2 m \mathrm{~L}} \leqq t \leqq \frac{m x+2 j \mathrm{~L}}{2 m \mathrm{~L}} .
\end{aligned}
$$

These equations are to be formed for

$$
j=1,2, \ldots m-2 i-1,
$$

and then the first of thom, formed for $j=m-2 i$ is to be super- 
added-namely

$$
\begin{aligned}
y=\mathrm{A} x\left(\frac{\mathrm{T}}{2}-t\right)+\mathrm{B}( & \left(x-\frac{2 i \mathrm{~L}}{m}\right)\left(\frac{m-i+1}{m} \mathrm{~T}-t\right) \\
& \text { for } \frac{m x+2(m-2 i-1) \mathrm{L}}{2 m \mathrm{~L}} \leq \frac{t}{\mathrm{~T}} \leqq \frac{2 \mathrm{~L}-x}{2 \mathrm{~L}} .
\end{aligned}
$$

Accordingly the velocity of the descending motion fluctuates between the values

$$
-(\mathrm{A}+\mathrm{B}) x+\frac{2 i}{m} \mathrm{BL} \text { and }-(\mathrm{A}+\mathrm{B}) x+\frac{2 i+1}{m} \mathrm{BL} \text {. }
$$

The other half of the ascending motion now remains to be considered. It is represented by the equations

$$
\begin{array}{r}
y=-\mathrm{A}(\mathrm{L}-x)(\mathrm{T}-t)-\mathrm{B}\left(\frac{2 i+1}{m} \mathrm{~L}-x\right)\left(\frac{m-i-1+j}{m} \mathrm{~T}-t\right) \\
\quad \text { for } \frac{2(m+j-1) \mathrm{L}-m x}{2 m \mathrm{~L}} \leqq \frac{t}{\mathrm{~T}} \leqq \frac{2(m-2 i-1+j) \mathrm{L}+m x}{2 m \mathrm{~L}}, \\
y=-\mathrm{A}(\mathrm{L}-x)(\mathrm{T}-t)+\mathrm{B}\left(x-\frac{2 i}{m} \mathrm{~L}\right)\left(\frac{m-i+j+1}{m} \mathrm{~T}-t\right) \\
\quad \text { for } \frac{2(m+j-2 i-1) \mathrm{L}+m x}{2 m \mathrm{~L}} \leqq \frac{t}{\mathrm{~T}} \leqq \frac{2(m+j) \mathrm{L}-m x}{2 m \mathrm{~L}} .
\end{array}
$$

These equations are to be formed for $j=1,2, \ldots 2 i-1$. The first of them holds good also for $j=2 i$; if the latter has been superadded, the entire system concludes with the equation

$$
\begin{array}{r}
y=-\mathrm{A}(\mathrm{L}-x)(\mathrm{T}-t)+\mathrm{B}\left(x-\frac{2 i}{m} \mathrm{~L}\right)\left(\frac{m+i+1}{m} \mathrm{~T}-t\right) \\
\quad \text { for } \frac{2(m-1) \mathrm{L}+m x}{2 m \mathrm{~L}} \leqq t \leqq 1 .
\end{array}
$$

Analogous formula can be constructed for those parts of the string which are limited by the points $x=\frac{2 i-1}{m} \mathrm{~L}$ and $x=\frac{2 i}{m} \mathrm{~L}$. This, however, shall not here be pursued further.

18. The function $y$ defined by the foregoing conducts to a very simple Fourrier series. We find, namely,

$$
y=\Sigma \mathrm{S}_{n} \sin \frac{2 n \pi t}{\mathrm{~T}}=\eta+\Delta \eta
$$

if $\eta$ and $\Delta \eta$ have the signification stated in no. 16 , so that $\eta$ is equal to the series given in (31), while, according to (34),

$$
\Delta \eta=\frac{\mathrm{BLT}}{m^{2} \pi^{2}} \sum \frac{1}{n^{2}} \sin \frac{n m \pi x}{\mathrm{~L}}-\sin \frac{2 n m \pi t}{\mathrm{~T}} .
$$



have

or

Both series can be integrated term by term; hence we

$$
\mathrm{S}_{n}=\frac{2}{\mathrm{~T}} \int_{0}^{\mathrm{T}}(\eta+\Delta \eta) d t=\frac{(\mathrm{A}+\mathrm{B}) \mathrm{LT}}{n^{2} \pi^{2}} \sin \frac{n \pi x}{\mathrm{~L}}
$$

$$
=\frac{\mathrm{AL} T}{n^{2} \pi^{2}} \sin \frac{n \pi x}{\mathrm{~L}}
$$

according as $n$ is or is not divisible by $m$.

The representation of $y$ by a Fourrier series gives therefore

$$
y=\frac{\mathrm{LT}}{\pi^{2}} \Sigma \frac{\mathrm{A}_{n}}{n^{2}} \sin \frac{n \pi x}{\mathrm{~L}} \sin \frac{2 n \pi t}{\mathrm{~T}},
$$

in which, when $n$ is not divisible by $m$,

$$
\mathrm{A}_{n}=\mathrm{A} \text {, }
$$

and, when $n$ is divisible by $m$,

$$
\mathrm{A}_{n}=\mathrm{A}+\mathrm{B} \text {. }
$$

Christoffel's conditions of discontinuity are spontaneously fulfilled by the equations formed in no. 17; hence the quantities $A$ and $B$ are at first arbitrary. Observations have nevertheless shown that the upper tones corresponding to the places $x=\frac{\mathrm{L}}{m}, \frac{2 \mathrm{~L}}{m}, \ldots$ are not present in the sound of the string. $\mathrm{B}$ must therefore be $=-\mathrm{A}$.

Consequently according to no. 16 , for a point between $\mathrm{x}=0$ and $\mathrm{x}-\frac{\mathrm{I}}{\mathrm{m}}$, the velocity of the ascending motion is equal to $\mathrm{A} \frac{\mathrm{m}-1}{\mathrm{~m}}$, therefore independent of $\mathrm{x}$; but that of the descending motion fuctuates between the values

$$
0,-\mathrm{A} \frac{\mathrm{L}}{m}, 0,-\mathrm{A} \frac{\mathrm{L}}{m}, \ldots
$$

The curve of the velocities will therefore consist of a rectilinear ascending* and a scalariform descending portion.

This is in accordance with Helmholtz's figures (l. c.p. 144). In the declinations published by Neumann $(l . c$.$) the descend-$ ing portion is not scalariform, but slightly rippled, and thus the equation $\mathrm{A}+\mathrm{B}=0$ is not perfectly fulfilled. Indeed all the upper tones are absent only when the bow is drawn very uniformly across the string, and touches it very accurately at the wished-for node-point. The number of the ripples which p. 136.

* This is confirmed also by Clifton's observations: of. Donkin, $l . c$. 
220 Forms of the Vibrations of twitched and stroked Strings.

appear in Neumann's drawings agrees with the formulæ given in no. $16^{*}$.

For other points of the string both the descending and the ascending portion of the velocity-curve are rippledt. For $\frac{2 i}{m} \mathrm{~L} \leqq x \leqq \frac{2 i+\mathrm{L}}{m} \mathrm{~L}$ for example, the velocity of the ascending motion fluctuates, according to no. 17 , when $B=-A$, between the values

$$
\mathrm{AL} \frac{m-2 i-1}{m} \text { and } \mathrm{AL} \frac{m-2 i}{m} \text {, }
$$

that of the descending motion between

$$
-\mathrm{AL} \frac{2 i}{m} \text { and }-\mathrm{AL} \frac{2 i+1}{m} \text {. }
$$

If the vibration-form considered be resolved into two elementary vibrations $\eta$ and $\Delta \eta$ (conf. no. 16), and the amplitude of the central point of the string for the motion $\eta$ be denoted by $P$, while the amplitude of the central point of any one part of the string for the component $\Delta \eta$ is denoted by $\mathrm{P}^{\prime}$, we shall have, according to (28),

$$
\mathrm{A}=\frac{8 \mathrm{P}}{\mathrm{L} T}, \quad \mathrm{~B}=\frac{8 m^{2} \mathrm{P}^{\prime}}{\mathrm{L} \mathrm{T}},
$$

and therefore, when $B=-A$,

$$
\mathrm{P}^{\prime}=-\frac{\mathrm{P}}{m^{2}}
$$

The form of the string at a given time will be approximately the same as in the case described in no. 13, except that for the straight lines there occurring slightly rippled lines are to be substituted.

19. We may remark that Helmholtz gives the following two equations in place of the three equations (27):-

$$
\begin{aligned}
& y=\frac{8 \mathrm{P}}{\mathrm{LT}}(\mathrm{L}-x) t \text { for } 0 \leqq t \leqq \frac{x \mathrm{~T}}{\mathrm{~L}} ; \\
& y=\frac{8 \mathrm{P}}{\mathrm{LT}} x(\mathrm{~T}-t) \quad y \quad \frac{x \mathrm{~T}}{\mathrm{~L}} \leqq t \leqq \mathrm{~T} .
\end{aligned}
$$

They only agree with the first two of the equations (27) if * Disregarding isolated cases in which larger secondary ripples appear,
and thus the motion is still more complicated.

+ These ripplings are recognized also in the following manner:-If a bright violin-string vibrates over a dark ground, the field of vibration appears to the naked eye uniformly bright; but if observed by means of a lens, there appear in it a number of brighter lines running parallel with the string. 
$\frac{T}{2}$ is substituted for $\mathrm{T}$ and $\frac{\mathrm{P}}{2}$ for $\mathrm{P}$; and then they represent only one half of the motion, while $\mathrm{T}$ signifies the entire duration of a vibration. On the other hand, the trigonometric series given by (31) agrees in substance with the series found by Helmboltz, if', in the latter, $\mathfrak{T}$ is understood as denoting the time of the ascending motion of a single determined point of the string. How this series in Helmholtz's work is obtained does not appear to me to be clearly shown. For, apparently, the letters $x$ and $\mathfrak{I}$ are at one time used for a variable, and then again for a fixed point of the string; and it does not distinctly appear whether the mathematical development rests upon the assumption of the law enunciated in no. 8 as valid for all points of the string, or whether it is presupposed for a single point only. The latter is done by Donkin and Lord Rayleigh. But if the problem is to be determined, not only must $y$ but also $\frac{\partial y}{\partial x}$ be given as a function of $t$ for the one point of the string. This uncertainty asserts itself in tho works mentioned by rendering it necessary, in the determination of $\mathfrak{I}$ as a function of $x$, to go back to the observations.

Freiburg i. B., July 12, 1879.

XXVIII. Cyclones of the Bay of Bengal*. By W. R. BIRT. $T \mathrm{~T}$ is well known that the Bay of Bengal is a very dangerous 1 locality for our East-Indian shipping, especially the head of the Bay in the neighbourhood of Calcutta. The danger principally arises from the cyclones which sweep over the Bay, wrecking ships and raising immense storm-waves exceedingly destructive of human life. Thus in the year 1737, October 7 , three hundred thousand people are stated to have been drowned; more than one hundred years later, in 1876, October 29 to November 1, one hundred thousand people were drowned in Backergunge. In June 1822, fifty thousand people perished by the storm-wave. In the year 1864, October 2 to 5 , forty-eight thousand people were drowned in the Hooghly by the storm-wave; and in 1831, October 31, ten thousand people were drowned by the storm-wave. In the following year, 1832, May 21, between eight and ten thousand people perished by the same agency. The total loss of human life, as recorded in connexion with severe cyclones that swept over the Bay on the dates given above, is no less

* Report of the Madras Cyclone of May 1877, by J. Eliot, Esq, M.A., Meteorological Reporter to the Government of Bengal. Calcutta, 1879. Plil. Mag. S. 5. Vol. 9. No. 55. March $1880 . \quad$ R 